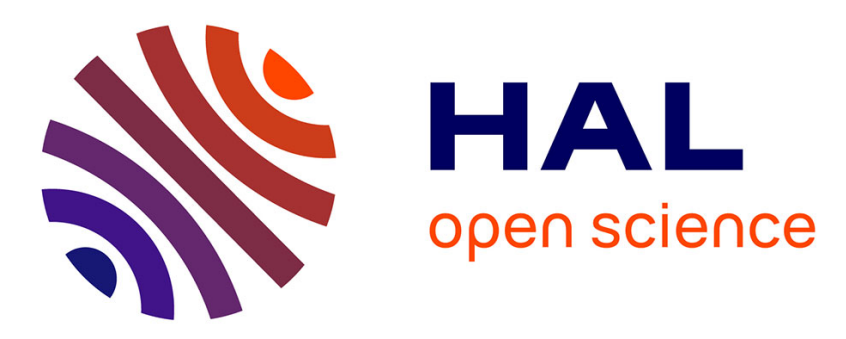

\title{
Skull conductivity estimation for EEG source localization
}

Facundo Costa, Hadj Batatia, Thomas Oberlin, Jean-Yves Tourneret

\section{To cite this version:}

Facundo Costa, Hadj Batatia, Thomas Oberlin, Jean-Yves Tourneret. Skull conductivity estimation for EEG source localization. IEEE Signal Processing Letters, 2017, vol.24 (nº), pp. 422-426. 10.1109/LSP.2017.2669101 . hal-01681070

\section{HAL Id: hal-01681070 https://hal.science/hal-01681070}

Submitted on 11 Jan 2018

HAL is a multi-disciplinary open access archive for the deposit and dissemination of scientific research documents, whether they are published or not. The documents may come from teaching and research institutions in France or abroad, or from public or private research centers.
L'archive ouverte pluridisciplinaire HAL, est destinée au dépôt et à la diffusion de documents scientifiques de niveau recherche, publiés ou non, émanant des établissements d'enseignement et de recherche français ou étrangers, des laboratoires publics ou privés. 


\section{Open Archive TOULOUSE Archive Ouverte (OATAO)}

OATAO is an open access repository that collects the work of Toulouse researchers and makes it freely available over the web where possible.

This is an author-deposited version published in : http://oatao.univ-toulouse.fr/ Eprints ID : 19375

To link to this article : DOI: 10.1109/LSP.2017.2669101

URL : ttp://dx.doi.org/10.1109/LSP.2017.2669101

To cite this version : Costa, Facundo hernan and Batatia, Hadj and Oberlin, Thomas and Tourneret, Jean-Yves Skull conductivity estimation for EEG source localization. (2017) IEEE Signal Processing Letters, vol.24 ( $\left.{ }^{\circ} 4\right)$. pp. 422-426. ISSN 1070-9908

Any correspondence concerning this service should be sent to the repository administrator: staff-oatao@ listes-diff.inp-toulouse.fr 


\title{
Skull Conductivity Estimation for EEG Source Localization
}

\author{
Facundo Costa, Hadj Batatia, Thomas Oberlin, and Jean-Yves Tourneret
}

\begin{abstract}
A reliable leadfield matrix is needed to solve the magnetoencephalography/electroencephalography (M/EEG) source localization problem. The computation of this matrix requires several physical parameters, including the conductivity of the tissues that compose the subject's head. Since it is not precisely known, we modify a recent Bayesian algorithm to estimate the skull conductivity jointly with the brain activity directly from the M/EEG measurements. Synthetic and real data are used to compare our technique with two optimization algorithms, showing that the proposed method is able to provide results of similar or better quality with the advantage of being applicable in a more general case.
\end{abstract}

Index Terms-Bayes methods, M/EEG measurements, source localization, sparsity.

\section{INTRODUCTION}

$\mathbf{M}$ AGNETOENCEPHALOGRAPHY/electroencephalography (M/EEG) source localization has been receiving an increasing amount of interest in the signal processing literature in the last decade. One of the most common models used to solve the associated ill-posed problem is the distributed source model [1], [2], which expresses the relationship between the brain activity and the M/EEG measurements by the leadfield matrix.

The leadfield matrix depends on the geometry and composition of the subject's head, most noticeably on the conductivities of the different tissues. Most of the tissue conductivities have values that are well accepted in the literature [3]. However, there has been some controversy regarding the conductivity of the human skull [2] with authors reporting values that differ in almost an order of magnitude [4], [5]. Most of the source localization techniques use a default value for this parameter. However, this value can affect the reconstruction considerably [3], [6]-[8] and has been shown to vary significantly across different subjects [2], [9]. To increase the reconstruction quality without measuring the skull conductivity directly, several methods try to estimate it jointly with the brain activity directly from the M/EEG measurements [10]-[17]. However, most of them require very restrictive conditions to yield good results. Assumptions required by the existing methods to estimate the skull conductivity include a very good a priori knowledge

Manuscript received October 16, 2016; revised January 5, 2017; accepted February 10, 2017. Date of publication February 14, 2017; date of current version March 3, 2017. The associate editor coordinating the review of this manuscript and approving it for publication was Dr. Charles Kervrann. (Corresponding author: J.-Y. Tourneret.)

The authors are with INP/ENSEEIHT, University of Toulouse, 31071 Toulouse, France (e-mail: facundo.costa@enseeiht.fr; hadj.batatia@enseeiht.fr; thomas.oberlin@enseeiht.fr; jean-yves.tourneret@enseeiht.fr).

Color versions of one or more of the figures in this letter are available online at http://ieeexplore.ieee.org.

Digital Object Identifier 10.1109/LSP.2017.2669101 about the active dipole positions [10], [11], the presence of only one active dipole [12], or the existence of few possible discrete values for the skull conductivity [13], [14].

In previous works [18], [19], we introduced a hierarchical Bayesian model to promote structured sparsity for recovering point-like brain activity. In the present letter (and the associated technical report [20]), we generalize this model to estimate the skull conductivity jointly with the brain activity in a completely unsupervised framework. A Markov chain Monte Carlo (MCMC) technique is investigated to generate samples asymptotically distributed according to the posterior distribution of this model. These samples are then used to build estimators of the model parameters including the skull conductivity of the brain.

This letter is organized as follows. The proposed inverse problem for EEG source localization and its associated Bayesian model are introduced in Section II. Section III presents the MCMC method (more precisely, a partially collapsed Gibbs sampler) used to sample the posterior distribution of this model. Section IV contains the main contribution of this letter, namely the introduction of a parametric model relating the leadfield matrix to the skull conductivity. Experiments conducted on synthetic and real data are presented in Section V. Conclusions are finally reported in Section VI.

\section{Proposed Hierarchical Bayesian Model}

The M/EEG inverse problem can be formulated as

$$
\boldsymbol{Y}=\boldsymbol{H}(\rho) \boldsymbol{X}+\boldsymbol{E}
$$

where the amplitudes of the $N$ dipoles in the $T$ time samples are concatenated in the matrix $\boldsymbol{X}=\left[\boldsymbol{x}^{1}, \ldots, \boldsymbol{x}^{T}\right] \in \mathbb{R}^{N \times T}$, $\boldsymbol{H}(\rho) \in \mathbb{R}^{M \times N}$ is the lead field operator that depends on the skull conductivity $\rho$ (with its columns normalized to compensate the depth biasing effect [1]), $\boldsymbol{Y}=\left[\boldsymbol{y}^{1}, \ldots, \boldsymbol{y}^{T}\right] \in \mathbb{R}^{M \times T}$ contains the measurements of the $M$ electrodes, and $\boldsymbol{E}=$ $\left[\boldsymbol{e}^{1}, \ldots, \boldsymbol{e}^{T}\right] \in \mathbb{R}^{M \times T}$ is the measurement noise matrix. The problem addressed in this work is the joint estimation of $\boldsymbol{X}$ and $\rho$ from the measurements $\boldsymbol{Y}$ and the function $\boldsymbol{H}(\rho)$.

\section{A. Likelihood}

We assume an independent white Gaussian noise with a variance $\sigma_{n}^{2}$, which leads to the following Gaussian likelihood:

$$
f\left(\boldsymbol{Y} \mid \boldsymbol{X}, \sigma_{n}^{2}, \rho\right)=\prod_{t=1}^{T} \mathcal{N}\left(\boldsymbol{y}^{t} \mid \boldsymbol{H}(\rho) \boldsymbol{x}^{t}, \sigma_{n}^{2} \mathbb{I}_{M}\right)
$$

where $\mathcal{N}$ is the Gaussian distribution, $\boldsymbol{m}^{j}$ represents the $j$ th column of $M$, and $\mathbb{I}_{M}$ is the $M \times M$ identity matrix. Note that if the noise is not white, the noise covariance can be estimated from the measurements and used to whiten the data [21]. 
TABLE I

Prior Distributions of THE PRoposed Model $f\left(z_{i} \mid \omega\right), f\left(\tau_{i}^{2} \mid a\right)$, $f\left(\boldsymbol{x}_{i} \mid z_{i}, \tau_{i}^{2}, \sigma_{n}^{2}\right), f\left(\sigma_{n}^{2}\right), f(a)$, AND $f(\omega)$

\begin{tabular}{cc}
\hline \hline$z_{i}$ & $\mathcal{B}\left(z_{i} \mid \omega\right)$ \\
$\tau_{i}^{2}$ & $\mathcal{G}\left(\tau_{i}^{2} \mid \frac{T+1}{2}, \frac{a}{2}\right)$ \\
& $\delta\left(\boldsymbol{x}_{i}\right) \quad$ if $z_{i}=0$ \\
$\boldsymbol{x}_{i}$ & $\mathcal{N}\left(0, \sigma_{n}^{2} \tau_{i}^{2} \mathbb{I}_{T}\right)$ if $z_{i}=1$ \\
$\sigma_{n}^{2}$ & $\frac{1}{\sigma_{n}^{2}} 1_{\mathbb{R}^{+}}\left(\sigma_{n}^{2}\right)$ \\
$a$ & $\mathcal{G}(a \mid \alpha, \beta)$ \\
$\omega$ & $\mathcal{U}(\omega \mid 0,1)$ \\
\hline \hline
\end{tabular}

\section{B. Prior Distributions}

This section generalizes the hierarchical Bayesian model of [18] to include the skull conductivity. The priors used for each of the model parameters and hyperparameters (except for $\rho$ ) are the same as were used in [18] and are summarized in Table I, where $\mathcal{B}, \mathcal{G}$, and $\mathcal{U}$ stand for the Bernoulli, gamma, and uniform distributions, respectively, $\delta$ is the Dirac delta function, $\boldsymbol{m}_{i}$ denotes the $i$ th row of $M, 1_{\mathbb{R}^{+}}(x)=1$ is the indicator function on $\mathbb{R}^{+}$, and $\alpha=\beta=1$. In this table, we can see that each row $\boldsymbol{x}_{i}$ is either jointly zero or nonzero, which is indicated by the discrete variable $z_{i} . \omega$ is a variable between 0 and 1 that indicates the probability of having $z_{i}=1$, whereas $a$ controls the amplitude of the nonzero rows of $\boldsymbol{X}$. Note that $\tau^{2}$ is a latent variable that is only introduced to accelerate the convergence of the algorithm. Finally, the noise variance $\sigma_{n}^{2}$ is assigned a Jeffrey's prior reflecting the absence of knowledge about this parameter. The reader is invited to consult [18] for more motivations and details about the Bayesian model used for EEG source localization.

1) Skull Conductivity: We assign a noninformative uniform prior for $\rho$

$$
f(\rho)=\mathcal{U}\left(\rho \mid \rho_{\min }, \rho_{\max }\right) .
$$

To select the range of this uniform distribution, we consider an interval containing the conductivities reported in the literature [4], [5], i.e., $\rho_{\min }=3.3 \frac{\mathrm{m} S}{\mathrm{~m}}$ and $\rho_{\max }=33 \frac{\mathrm{m} S}{\mathrm{~m}}$. This choice results in scalp to skull conductivity ratios inside $[10,100]$.

\section{Posterior Distribution}

Denoting the unknown parameter vector $\boldsymbol{\theta}=$ $\left\{\boldsymbol{X}, \sigma_{n}^{2}, \rho, \boldsymbol{z}, \boldsymbol{\tau}^{2}, \omega, a\right\}$, the posterior distribution is

$$
\begin{gathered}
f(\boldsymbol{\theta} \mid \boldsymbol{Y}) \propto f\left(\boldsymbol{Y} \mid \boldsymbol{X}, \sigma_{n}^{2}, \rho\right) f\left(\boldsymbol{X} \mid \boldsymbol{\tau}^{2}, \boldsymbol{z}, \sigma_{n}^{2}\right) f(\boldsymbol{z} \mid \omega) \\
f\left(\boldsymbol{\tau}^{2} \mid a\right) f\left(\sigma_{n}^{2}\right) f(a) f(\omega) f(\rho) .
\end{gathered}
$$

Unfortunately, the posterior (4) is too complex to express the estimators of the model parameters and hyperparameters in closed form. As a consequence, we propose to draw samples from (4) and use them to build estimators of the brain activity, the skull conductivity, and the model hyperparameters. The proposed sampler is presented in the next section.

\section{Partially Collapsed GibBs SAmpler}

We investigate a partially collapsed Gibbs sampler (see [19] for motivations) that generates samples asymptotically distributed according to (4). Denoting as $\boldsymbol{X}_{-i}$ the matrix $\boldsymbol{X}$ whose $i$ th row has been replaced by zeros, the proposed sampler generates samples of the different variables according to their

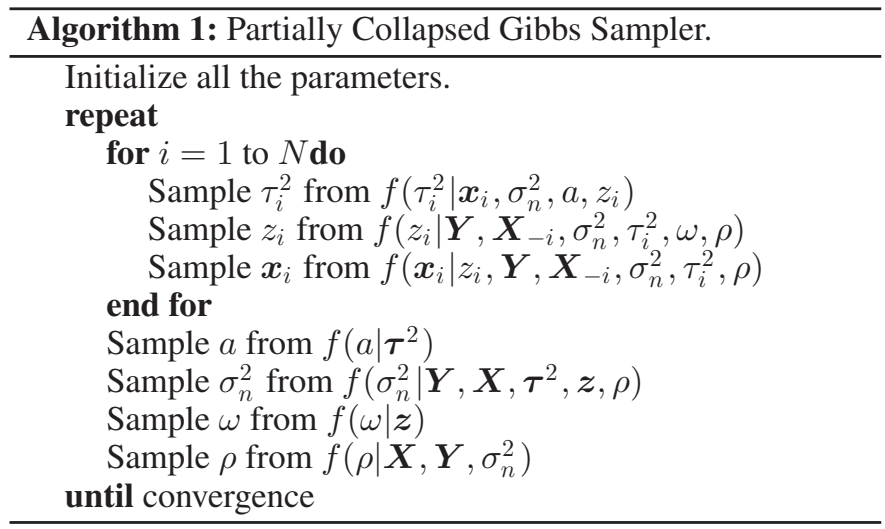

conditional distributions, as shown in Algorithm 1. The corresponding conditional distributions can be found in [19], except for the distribution of $\rho$ that is detailed below.

The conditional distribution of the skull conductivity is

$f\left(\rho \mid \boldsymbol{X}, \boldsymbol{Y}, \sigma_{n}^{2}\right) \propto \exp \left(-\frac{\|\boldsymbol{H}(\rho) \boldsymbol{X}-\boldsymbol{Y}\|_{F}^{2}}{2 \sigma_{n}^{2}}\right) 1_{\left[\rho_{\min }, \rho_{\mathrm{max}}\right]}(\rho)$.

The following section explains how to efficiently model the operator $\boldsymbol{H}(\rho)$ and how to sample from (5).

\section{LEADFIELD MATRIX MODEL}

\section{A. Approximation Using a Polynomial Matrix}

The complexity of the operator $\boldsymbol{H}(\rho)$ makes sampling from $f\left(\rho \mid \boldsymbol{X}, \boldsymbol{Y}, \sigma_{n}^{2}\right)$ difficult for both realistic and spherical head models. To simplify this dependence, Şengül and Baysal [11] proposed to use a linear approximation of $\boldsymbol{H}\left(\rho_{k}\right)$ around a local value $\rho_{k}$. Instead, we propose to approximate $\boldsymbol{H}(\rho)$ on the whole range $\left[\rho_{\min }, \rho_{\max }\right]$ by using a polynomial matrix of degree $L$ denoted by $\hat{\boldsymbol{H}}_{L}(\rho)$. Each element of $\hat{\boldsymbol{H}}_{L}(\rho)$ is defined as $\hat{h}_{i, j}(\rho)=\sum_{l=0}^{L} c_{i, j, l} \rho^{l}$. The values of the coefficients $c_{i, j, l}$ will be estimated by applying polynomial least-squares fitting to the exact values of $\boldsymbol{H}\left(\rho_{k}\right)$ for $\rho_{k}=\rho_{\min }+\frac{k\left(\rho_{\max }-\rho_{\min }\right)}{K-1}$ with $k=0, \ldots, K-1$ and the approximating polynomial matrix $\hat{\boldsymbol{H}}_{L, K}(\rho)$ (see [20] for more details).

\section{B. Sampling the Skull Conductivity}

Approximating the relationship between the operator and the skull conductivity with a polynomial matrix allows us to have a simple closed-form expression for $f\left(\rho \mid \boldsymbol{X}, \boldsymbol{Y}, \sigma_{n}^{2}\right)$

$$
f\left(\rho \mid \boldsymbol{X}, \boldsymbol{Y}, \sigma_{n}^{2}\right) \propto \exp [-g(\rho)] 1_{\left[\rho_{\mathrm{min}}, \rho_{\mathrm{max}}\right]}(\rho)
$$

where $g(\rho)=\frac{1}{2 \sigma_{n}^{2}}\left\|\hat{\boldsymbol{H}}_{L, K}(\rho) \boldsymbol{X}-\boldsymbol{Y}\right\|_{F}^{2}$ is a polynomial of order $2 L$. To sample from (6), we adopt a Metropolis-Hastings scheme [22]. More precisely, at each iteration, we propose to generate a new sample $\rho_{\text {prop }}=\rho_{\text {old }}+\epsilon$ using a random walk. This sample is then accepted with the probability

$$
P_{a}=\min \left(\frac{f\left(\rho_{\text {prop }} \mid \boldsymbol{X}, \boldsymbol{Y}, \sigma_{n}^{2}\right)}{f\left(\rho_{\text {old }} \mid \boldsymbol{X}, \boldsymbol{Y}, \sigma_{n}^{2}\right)}, 1\right) .
$$

For the random walk, we propose to use a Gaussian distribution for $\epsilon$, i.e., $f(\epsilon)=\mathcal{N}\left(0, \sigma_{\epsilon}^{2}\right)$, where $\sigma_{\epsilon}^{2}$ is adjusted by cross validation in order to obtain an appropriate acceptance rate, as recommended in [22]. 


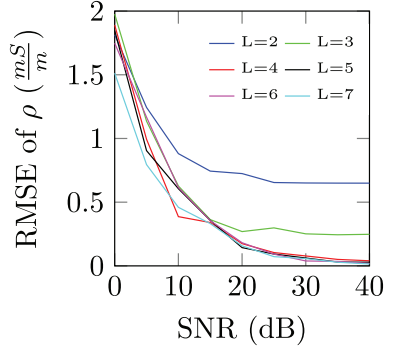

(a)

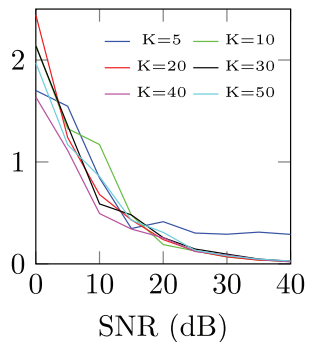

(b)
Fig. 1. RMSE of the skull conductivity estimation versus SNR for different values of $L$ and $K$. (a) RMSE of $\rho$ versus SNR ( $K=100$ ). (b) RMSE of $\rho$ versus $\operatorname{SNR}(L=7)$.

\section{Proposals}

As mentioned in [18], the sampler presented above may get stuck around local maxima of the target distribution. Multiple-dipole shift (MDS) and interchain (IC) proposals for the dipoles location were introduced in [18] to solve this issue. The MDS proposal moves random nonzeros to neighboring positions, whereas the IC proposal allows different MCMC chains to exchange their dipole positions. These moves are then accepted or rejected using the classical Metropolis-Hastings acceptance rate [22]. In order to build more efficient proposals, we propose to jointly move the dipole locations and skull conductivity (see [20] for more details).

\section{EXPERIMENTAL RESULTS}

\section{A. Synthetic Data}

Synthetic data are first considered using a 200-dipole Stok four-sphere head model [23] with 41 electrodes. The values of $L$ (degree of the polynomial) and $K$ (number points in the leastsquare fitting) need to be adjusted to build an accurate estimator $\hat{\boldsymbol{H}}_{L, K}(\rho)$. For that purpose, the value $\hat{\rho}$ that minimizes (6) was calculated for ten different ground truth values of the skull conductivity $\rho_{\mathrm{gt}}$ and 200 different values of $\boldsymbol{X}$ for different values of $L$ and $K$. The root-mean-square errors (RMSE) between $\rho_{\mathrm{gt}}$ and their estimations $\hat{\rho}$ are displayed in Fig. 1 (Fig. 1(a) corresponds to $K=100$ and different values of $L$, whereas Fig. 1(b) was obtained with $L=7$ and different values of $K$ ). Increasing the values of $L$ and $K$ reduces the estimation RMSEs. However, this effect is limited by the amount of noise affecting the EEG measurements. Thus, for common values of signal-to-noise ratio (SNR), choosing very high values of $L$ and $K$ does not improve the estimation of $\rho$. Based on these results, we used $L=4$ and $K=10$ in all experiments. The next sections investigate two different kinds of activations: 1) a single-dipole activation and 2) multiple-dipole activations.

1) Single-Dipole Activation: The first experiment considers a single random dipole with a damped sine activation of $5 \mathrm{~Hz}$ sampled at $200 \mathrm{~Hz}$. The operator was calculated for $\rho_{\mathrm{gt}}=3.6 \mathrm{mS} / \mathrm{m}$, and white noise was added to obtain $\mathrm{SNR}=10 \mathrm{~dB}$. The proposed method is compared with two other approaches: 1) our previous model [18] with a default value $\rho_{\mathrm{fix}}=\frac{\rho_{\mathrm{max}}+\rho_{\mathrm{min}}}{2}=18.15 \mathrm{mS} / \mathrm{m}$; and 2) the optimization method studied by Vallaghé et al. [12] that is able to estimate $\rho$ and the brain activity jointly since there is only one active dipole for this example.

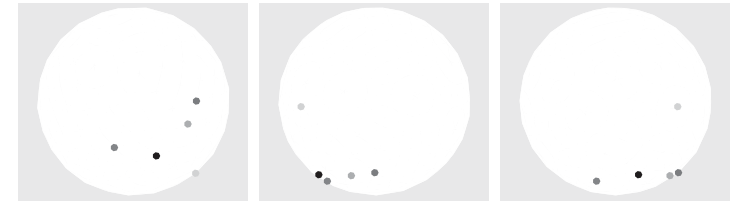

(a)

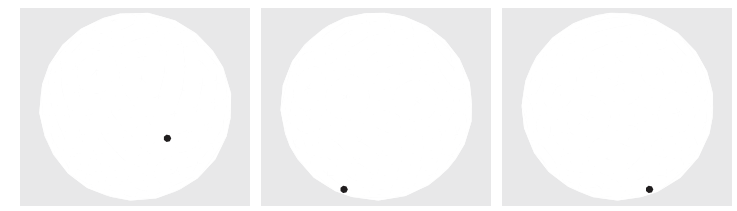

(b)

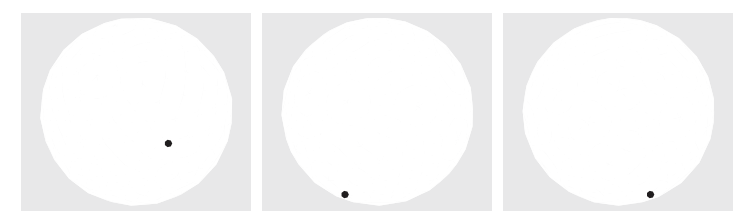

(c)

Fig. 2. Estimated activity for the single-dipole simulation, axial, coronal, and sagittal views, respectively. (a) Default- $\rho$ model. (b) Proposed method. (c) Vallaghé's method.

TABLE II

ERROR METRICS FOR DifFERENT PARAMETERS IN THE SINGLE-DIPOLE SYNTHETIC EXPERIMENT

\begin{tabular}{lccc}
\hline \hline Method & $\left\|\hat{X}-X_{\mathrm{gt}}\right\|_{F}^{2}$ & $\left|\hat{\rho}-\rho_{\mathrm{gt}}\right|$ & $\frac{\left\|\boldsymbol{H}\left(\rho_{\mathrm{gt}}\right)-\boldsymbol{H}(\hat{\rho})\right\|_{F}^{2}}{\left\|X_{\mathrm{gt}}\right\|_{F}^{2}}$ \\
\hline Default $\rho$ & & \\
Vallaghé & 1.36 & $1.46 \times 10^{-2}$ & $7.58 \times 10^{-2}$ \\
Proposed Method & $6.17 \times 10^{-3}$ & $2.65 \times 10^{-4}$ & $9.23 \times 10^{-5}$ \\
\hline \hline
\end{tabular}

The estimated dipole locations are shown in Fig. 2. We can see that the model of [18] with $\rho=\rho_{\text {fix }}$ fails to recover the dipole location and spreads the activity over a significant area of the brain, illustrating the loss of reconstruction performance when the wrong value of $\rho$ is used. The optimization method is able to recover the dipole position correctly and gives an average estimate (computed using 20 Monte Carlo runs) of the skull conductivity $\hat{\rho}=3.85 \mathrm{mS} / \mathrm{m}$. The proposed method provides a mean value of the estimates equal to $3.49 \mathrm{mS} / \mathrm{m}$ with a standard deviation of $0.12 \mathrm{mS} / \mathrm{m}$. Table II complements these results by other quantitative results. Note that additional experiments conducted on synthetic data with deeper dipoles can also be found in the associated technical report [20].

The price to pay for the good performance of the proposed method is its computational complexity. Using MATLAB implementations in a modern Xeon CPU E3-1240 processor, each simulation was processed on average in $96.1 \mathrm{~s}$ by the proposed model, $51.29 \mathrm{~s}$ by the default $\rho$ model, and $23.8 \mathrm{~s}$ by Vallaghé's method.

2) Multiple-Dipole Activations: The second kind of experiments considers a variable amount of active dipoles. In each experiment, $C$ active dipoles were simulated with damped sinusoidal waves with frequencies varying between 5 and $20 \mathrm{~Hz}$ sampled at $200 \mathrm{~Hz}$. Twenty different simulations were conducted for each value of $C=1, \ldots, 16$, each one having a different set of active dipoles and a different value of $\rho_{\mathrm{gt}}$, resulting in a total 


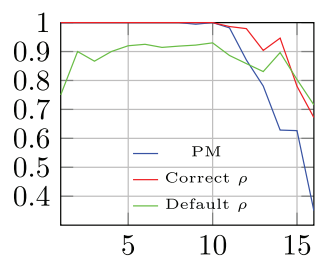

Amount of active dipoles

(a)

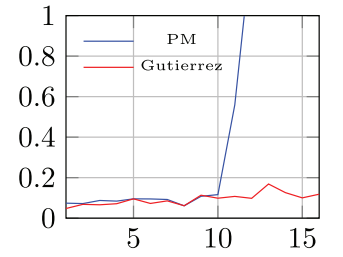

Amount of active dipoles

(b)
Fig. 3. Performance metrics for the multiple-dipole simulations as a function of $C$. (a) Recovery rate versus $C$. (b) RMSE of $\hat{\rho}$ estimation versus $C$.

of 320 experiments. Noise was added to the measurements to obtain $\mathrm{SNR}=30 \mathrm{~dB}$.

The proposed method was compared with: 1) the method of [18] using the correct value $\rho_{\mathrm{fix}}=\rho_{\mathrm{gt}}$; 2) the method of [18] with $\rho_{\text {fix }}=\frac{\rho_{\mathrm{min}}+\rho_{\mathrm{max}}}{2}=18.15 \mathrm{mS} / \mathrm{m}$; and 3) the method of Gutierrez [10]. Note that the method of [10] requires to know the locations of the active dipoles in advance.

We define the recovery rate as the proportion of active dipoles that are recovered by a given method. Fig. 3(a) displays the average recovery rate for the first three methods as a function of $C$ (Gutiérrez's method was not considered here since it knows the dipole locations in advance). For $C \leq 10$, the recovery rate of the proposed method is very close to the method that knows the correct value of $\rho$ and performs significantly better than the method of [18] running with the default value of $\rho$. Fig. 3(b) shows the RMSE of the estimator $\widehat{\rho}$ as a function of $C$ for the two methods allowing this estimation. For $C \leq 10$, the RMSE of the proposed estimator is very close to the one of Gutiérrez's method (that knows the active dipole positions in advance). To summarize, the performance of the proposed method is very satisfactory for $C \leq 10$. For $C>10$, this performance drops significantly.

\section{B. Real Data}

1) Auditory Evoked Responses: This section considers the left-ear auditory pure-tone stimulus data set from the MNE software [24], [25] to evaluate the proposed method. A realistic boundary element method head model with 1844 dipoles located on the cortex and oriented normally to the brain surface is available with this dataset. The data were sampled with 306 MEG sensors at $600 \mathrm{~Hz}$, low-pass filtered at $40 \mathrm{~Hz}$, and downsampled to $150 \mathrm{~Hz}$. The measurements corresponding to $200 \mathrm{~ms}$ of data preceding each stimulus were considered to estimate the noise covariance matrix that was used to whiten the measurements. Fifty-one epochs were averaged to calculate $\boldsymbol{Y}$. The activity of the source dipoles was estimated jointly with the skull conductivity for the period lasting $500 \mathrm{~ms}$ after the stimulus.

The proposed method was compared with 1) a default- $\rho$ model that uses $\rho=6 \mathrm{mS} / \mathrm{m}$ corresponding to a ratio between the scalp and skull conductivities equal to 50 (the default value used by the MNE software), and 2) the $\ell_{21}$ mixed-norm regularization introduced in [21] also using $\rho=6 \mathrm{mS} / \mathrm{m}$.

We can see in Fig. 4 that the three models find the brain activity focused on the auditory cortices. The main difference is that our method finds activity more focused in a lower amount of dipoles. In addition, our method results in $\hat{\rho}=10.6 \mathrm{mS} / \mathrm{m}$, corresponding to a ratio between the scalp and skull conductivities equal to 31 . This ratio is considerably lower than the value

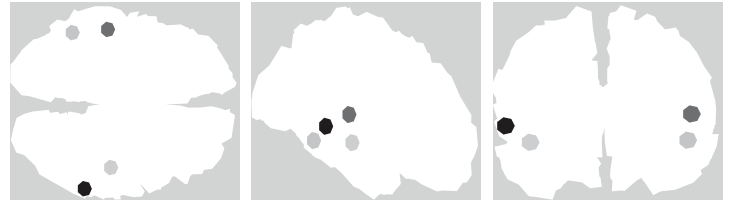

(a)

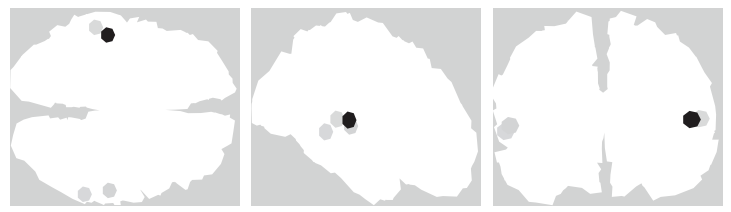

(b)

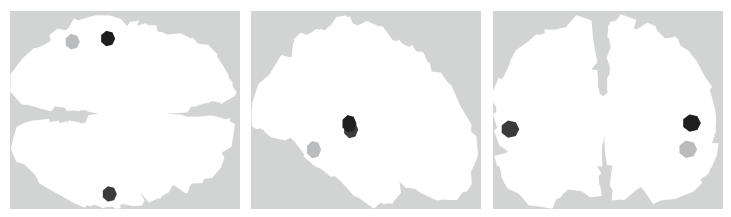

(c)

Fig. 4. Estimated activity for the auditory evoked responses. (a) Default- $\rho$ model. (b) $\ell_{21}$ mixed norm. (c) Proposed method.

of 80 used in the first studies [4] and closer to the value reported in more recent studies [5], [26].

\section{CONCLUSION}

This letter extended an existing hierarchical Bayesian model for sparse $\mathrm{M} / \mathrm{EEG}$ reconstruction to make it capable of estimating the skull conductivity jointly with the underlying brain activity. An MCMC method was used to generate samples asymptotically distributed according to the posterior distribution of this model. These samples were used to calculate the estimators of the model parameters and hyperparameters in a completely unsupervised framework. A polynomial approximation of the leadfield matrix was used to simplify the sampling of the skull conductivity. Synthetic data were used to compare the proposed method with the same model using a default value of the skull conductivity. The obtained results showed that the estimation of the skull conductivity improves the estimation of the underlying brain activity, especially in the case of multiple active dipoles. In addition, the proposed method was compared with the optimization techniques introduced by Vallaghe et al. [12] (that requires having only one dipole active) and Gutiérrez et al. [10] (that requires knowing the active dipole positions in advance). The proposed method showed very competitive results for these examples. Experiments conducted on an auditory evoked response real dataset showed that estimating the skull conductivity leads to a more focal result when compared to other sparse promoting models (such as the $\ell_{21}$ mixed-norm regularization). Moreover, the estimated value of the skull conductivity provided by the proposed method was close to the values reported by recent studies, which is a very interesting result. Future work includes the application of the proposed algorithm to other types of real data, including pure EEG and mixed $\mathrm{M} / \mathrm{EEG}$ measurements. Another possibility is trying to replace the MCMC sampling scheme by an approximate message passing step, in order to reduce the computational complexity of the method. 


\section{REFERENCES}

[1] R. Grech et al., "Review on solving the inverse problem in EEG source analysis," J. Neuroeng. Rehabil., vol. 4, pp. 5-25, 2008

[2] H. Hallez et al., "Review on solving the forward problem in EEG source analysis," J. Neuroeng. Rehabil., vol. 4, pp. 46-75, 2007.

[3] Z. A. Acar and S. Makeig, "Effects of forward model errors on EEG source localization," Brain Topography, vol. 26, no. 3, pp. 378-396, 2013.

[4] L. Geddes and L. Baker, "The specific resistance of biological materialA compendium of data for the biomedical engineer and physiologist," Med. Biol. Eng. Comput., vol. 5, no. 3, pp. 271-293, 1967.

[5] T. F. Oostendorp, J. Delbeke, and D. F. Stegeman, "The conductivity of the human skull: results of in vivo and in vitro measurements," IEEE Trans. Biomed. Eng., vol. 47, no. 11, pp. 1487-1492, Nov. 2000

[6] S. Vallaghé and M. Clerc, "A global sensitivity analysis of three-and fourlayer EEG conductivity models," IEEE Trans. Biomed. Eng., vol. 56, no. 4, pp. 988-995, Apr. 2009.

[7] B. Vanrumste, G. Van Hoey, R. Van de Walle, M. D'havé, I. Lemahieu, and P. Boon, "Dipole location errors in electroencephalogram source analysis due to volume conductor model errors," Med. Biol. Eng. Comput., vol. 38, no. 5, pp. 528-534, 2000.

[8] N. G. Gençer and C. E. Acar, "Sensitivity of EEG and MEG measurements to tissue conductivity," Phys. Med. Biol., vol. 49, no. 5, pp. 701-717, 2004.

[9] S. Gonçalves et al., "In vivo measurement of the brain and skull resistivities using an EIT-based method and realistic models for the head," IEEE Trans. Biomed. Eng., vol. 50, no. 6, pp. 754-767, Jun. 2003.

[10] D. Gutiérrez, A. Nehorai, and C. H. Muravchik, "Estimating brain conductivities and dipole source signals with EEG arrays," IEEE Trans. Biomed. Eng., vol. 51, no. 12, pp. 2113-2122, Dec. 2004.

[11] G. Şengül and U. Baysal, "An extended Kalman filtering approach for the estimation of human head tissue conductivities by using EEG data: A simulation study," Physiol. Meas., vol. 33, no. 4, 2012, Art. no. 571.

[12] S. Vallaghé, M. Clerc, and J.-M. Badier, "In vivo conductivity estimation using somatosensory evoked potentials and cortical constraint on the source," in Proc. IEEE 4th Int. Symp. Biomed. Imag., Washington, DC, USA, 2007, pp. 1036-1039.

[13] S. Lew, C. H. Wolters, A. Anwander, S. Makeig, and R. S. MacLeod, "Improved EEG source analysis using low-resolution conductivity estimation in a four-compartment finite element head model," Human Brain Mapping, vol. 30, no. 9, pp. 2862-2878, 2009.
[14] S. Lew, C. Wolters, A. Anwander, S. Makeig, and R. MacLeod, "Low resolution conductivity estimation to improve source localization," Int. Congr. Ser, vol. 1300, pp. 149-152, 2007.

[15] Z. A. Acar, C. E. Acar, and S. Makeig, "Simultaneous head tissue conductivity and EEG source location estimation," NeuroImage, vol. 124, pp. 168-180, 2016.

[16] U. Baysal and J. Haueisen, "Use of a priori information in estimating tissue resistivities-Application to human data in vivo," Physiol. Meas., vol. 25, no. 3, 2004, Art. no. 737.

[17] M.-X. Huang et al., "A novel integrated MEG and EEG analysis method for dipolar sources," Neuroimage, vol. 37, no. 3, pp. 731-748, 2007.

[18] F. Costa, H. Batatia, T. Oberlin, C. D'Giano, and J.-Y. Tourneret, "Bayesian EEG source localization using a structured sparsity prior," NeuroImage, vol. 144, pp. 142-152, Jan. 2017.

[19] F. Costa, H. Batatia, T. Oberlin, and J.-Y. Tourneret, "Bayesian structured sparsity priors for EEG source localization technical report," ENSEEIHT, Univ. Toulouse, Toulouse, France, Tech. Rep., 2015. [Online]. Available: http://arxiv.org/abs/1509.04576

[20] F. Costa, H. Batatia, T. Oberlin, and J.-Y. Tourneret, "EEG reconstruction and skull conductivity estimation using a Bayesian model promoting structured sparsity," IRIT/INP-ENSEEIHT, Univ. Toulouse, Toulouse, France, Tech. Rep., 2016. [Online]. Available: http://arxiv.org/abs/1609.06874

[21] A. Gramfort, M. Kowalski, and M. Hämäläinen, "Mixed-norm estimates for the M/EEG inverse problem using accelerated gradient methods," Phys. Med. Biol., vol. 57, no. 7, pp. 1937-1961, 2012.

[22] G. Casella and C. P. Robert, Monte Carlo Statistical Methods. New York, NY, USA: Springer-Verlag, 1999.

[23] C. J. Stok, "The inverse problem in EEG and MEG with application to visual evoked responses," Ph.D. dissertation, Univ. Twente, Enschede, The Netherlands, 1986

[24] A. Gramfort et al., "MNE software for processing MEG and EEG data," Neurolmage, vol. 86, pp. 446-460, 2014.

[25] A. Gramfort et al., "MEG and EEG data analysis with MNE-Python," Frontiers Neurosci., vol. 7, no. 267, pp. 1-13, 2013.

[26] R. Hoekema et al., "Measurement of the conductivity of skull, temporarily removed during epilepsy surgery," Brain Topography, vol. 16, no. 1, pp. 29-38, 2003. 